\title{
MS34-01 | Towards the Design Of Molecular Materials
}

Brandenburg, Gerit (Heidelberg University, Heidelberg, GER)

New technologies are made possible by new materials, and until recently new materials could only be discovered experimentally. However, quantum mechanical approaches are now integrated to many design initiatives in academia and industry, underpinning efforts such as the computational crystal structure prediction (CSP [1]). The latest CSP blind test revealed two major remaining challenges:[2] (i) Dealing with a vast search space, in particular for molecules with increased flexibility. (ii) Crystal polymorphs are often separated by just a few kJ/mol, exceeding the accuracy ofdensity functional approximations (DFAs). Cost-effective electronic structure methods will be presented that gain up to four orders of magnitude in computational speed compared to traditional DFAs and are suited for optimizing a huge number of putative crystal structures [3]. Promising applications to the CSP of pharmaceutical-like molecules have been demonstrated recently [4]. On the other hand, recent developments in Quantum Monte-Carlo make it feasible to molecular crystals and we are now able to predict static lattice energies with potentially sub-chemical accuracy [5].

[1] S.L. Price, JGB, Molecular Crystal Structure Prediction; Elsevier Australia, 2017, 333-364.

[2] A.M. Reilly, et al. Acta. Cryst. B 2016, 72, 439.

[3] E. Caldeweyher, JGB, J. Phys.: Condens. Matter 2018, 30, 213001.

[4] L. Iuzzolino, P. McCabe, S.L. Price, JGB, Faraday Discuss. 2018, 211, 275.

[5] A. Zen, JGB, J. Klimeš, A. Tkatchenko, D. Alfè, A. Michaelides, Proc. Natl. Acad. Sci. USA 2018, $115,1724$. gerit-brandenburg.de 